\title{
Students' Motivation in Reading English text: A survey of Junior High
} School in Palembang

\author{
Depi Apriani \\ State University of Raden Fatah Palembang \\ depi@gmail.com \\ Manallulaili \\ State University of Raden Fatah Palembang \\ manallulaili_uin@radenpatah.ac.id \\ Renny Kurniasari \\ State University of Raden Fatah Palembang \\ rennykurniasari_uin@radenfatah.ac.id
}

\begin{abstract}
This study investigated students' reading motivation in English text. The Quantitative research methodology carried out in this study is in order to collect information or data that is donated. Researcher used a survey with descriptive analysis that is research whose source of data and information is mainly obtained from respondents as a research sample using questionnaires as an instrument of data collection to describe students reading motivation of junior high school in Palembang. This study was conducted at SMP N 7 Pemulutan with 104 as the participants taken from the nine grade students of that school by using simple random sampling. in collecting data, the researcher used Motivation Reading Questionnaire (MRQ). The questionnaire consisted of 53 items with eleven indicators. The result covers 1. Self efficacy can make students more motivated in reading. 2. Intrinsic factor included enjoyable and students' interest in reading. 3. Extrinsic factor covered such as for finishing assignment, for getting high getting praises, in getting high score.
\end{abstract}

Keywords: Students' reading motivation, English text, Self efficacy, Intrinsic motivation, Extrinsic motivation.

\section{A. Introduction}

Motivation is a great influence for students' in reading English text. McGeown (2013) states that motivation is crucial for students to develop their reading skill (p.2). Olsen (2017) also states that motivation is important because it explores how an increase in reading development. It can be said that without motivation the students tend to be lazy to learn especially in reading English text. 
Jadila: Journal of Development and Innovation

E-ISSN: 2723-6900

in Language and Literature Education

P-ISSN: 2745-9578

Publisher: Yayasan Karinosseff Muda Indonesi

Volume 1 Number 2, 2021

Page: $88-100$

In this study, the researcher observed reading motivation of students'

junior high school. This study similar to study conducted by Marhama (2013) stated that students' motivation was a big issue in the classroom. They are more engaged chatting with their friends than reading. The statement showed that the students have problems in reading motivation. Each student in that class may have different motivations in learning, especially in reading English, both intrinsically and extrinsically. It also can be seen as real phenomena toward teaching and learning process in the school based on the experience of the writer.

From the problems occurred above, in the consideration of theories discussed previously, the students' reading motivation are the cause of these problems. Thus, this study aims to investigate reading motivation of junior high school in Palembang.

\section{B. Literature Review}

\section{The Definition of Motivation}

Motivation is a process within a person that raises energy or enthusiasm to achieve something. According to Dornyei (2001) motivation refers to a conceptual, abstract theory that we use to describe why individuals think and behave as they do. Additionally, Brophy (2004) state that motives are hypothetical structures used to describe why individuals are doing things. For example, by going to a restaurant (strategy) to get food (goal), an individual responds to hunger (motive). In conclusion, motivation explains why people decide to do something, how hard they are going to persevere it and how long they are able to continue the operation.

This study there are two types of motivation intrinsic and extrinsic. Arnold (2000, p.14) intrinsic motivation refers to learning itself having its own reward, it means the learners are willingly and voluntary (not compulsorily) try to learn what they think it is worth or important for them. When students have intrinsic motivation, they are no negative impacts in having intrinsic motivation. On the other hand, Wimolmas (2012) also state 
Jadila: Journal of Development and Innovation

E-ISSN: 2723-6900

in Language and Literature Education

P-ISSN: 2745-9578

Publisher: Yayasan Karinosseff Muda Indonesi

Volume 1 Number 2, 2021

Page: $88-100$

that extrinsic motivation is based on external outcomes such as rewards and punishment. In short, intrinsic and extrinsic motivation are not suitable.

\section{The Concept of Reading}

Reading is a skill about understanding written text. According to Pang, Muaka, Bernbardt \& Kamil (2003) reading include of two related processes, first: word recognition indicate that a process of apprehend how one spoken language correspond to written symbols. Second: Comprehension is the process be understandable of words, sentence and connected text (p.6). Similarly, Cline, Jhonstone, \& King (2006) state that reading decodes and understand text for particular reader reasons, and readers interpret written text by converting text into words and translating it directly into context. In short reading is a skill of language to bring information from written text.

\section{The Concept of Reading Motivation}

In the field of education, reading motivation is the motivating urge to read an area of interest. According to Pang, Muaka, Bernbardt, and Kamil, (2003) a reader read a text to grasp its context and to use that information. The object of reading is closely linked to the desire of a person to read, to learn, to find knowledge, to be entertained, to individuals or as religious practice (p. 15). In addition, Wigfield, Guthrie, \& Perecevich (2010) state that used background knowledge during reading becomes standard with the text, has intrinsic and extrinsic motivation to read successfully. Hence, motivation to read as the personal interest, value, and beliefs of the subjects, methods and result of reading are individual.

\section{Motivation Theories Related Reading and Achievement Autonomy}

Autonomy allows students greater freedom and it is the choice that motives them. The relationship between autonomy and motivation is explained by Ryan \& Deci (as cited in Brophy,2004) encourage learners to 
Jadila: Journal of Development and Innovation

function as autonomous learners, research based on the theory of selfdetermination.

\section{Research Methodology}

The research methodology carried out in this study is in order to collect information or data that is donated. Researcher used a survey with descriptive analysis that is research whose source of data and information is mainly obtained from respondents as a research sample using questionnaires as an instrument of data collection to describe students reading motivation of junior high school in Palembang.

The participant of this study were the of SMP N 7 Pemulutan in the academic year 2020 2021. And to get the sample of this study, the sampling technique was probability sampling.

This study used motivation reading questionnaire (MRQ) from wigfield \& Guthrie (1997) for collecting data. Researcher distributed questionnaire to the nine grade students. The motivation reading questionnaire consisted of 53 item with eleven indicators. Questionnaire allowed a researcher to see the reading motivation of students from SMP N 7 Palembang by the source of data.

In analyzing the data, the researcher used descriptive survey analysis to gain students reading motivation of junior high school. In this study, the data was analyzed by identifying and interpreting the students reading motivation in English text from the questionnaires and described it by using percentage: $\mathrm{P}=\mathrm{F} / \mathrm{N} X 100 \%$.

\section{Results and Discussion}

\section{Results}

The findings of this study presented the research findings based on the results of reading motivation. the result of data analysis collected using a 
Jadila: Journal of Development and Innovation

questionnaire were presented in research conducted at junior high school by collecting data through questionnaire.

Table 1 Reading Efficacy

\begin{tabular}{|c|c|c|c|c|c|c|c|c|c|c|c|c|c|}
\hline \multirow[b]{2}{*}{ No } & \multirow[b]{2}{*}{ Statement } & \multicolumn{2}{|c|}{4} & \multicolumn{2}{|r|}{3} & \multicolumn{2}{|c|}{ Total } & \multicolumn{2}{|r|}{2} & \multicolumn{2}{|r|}{1} & \multicolumn{2}{|r|}{ Total } \\
\hline & & $\mathrm{F}$ & $\%$ & $\mathrm{~F}$ & $\%$ & $\mathbf{F}$ & $\%$ & $F$ & $\%$ & $F$ & $\%$ & $\mathbf{F}$ & $\%$ \\
\hline 1 & $\begin{array}{l}\text { I don't know } \\
\text { that I will do } \\
\text { well in reading } \\
\text { next year }\end{array}$ & 66 & $\begin{array}{c}63,4 \\
6\end{array}$ & 29 & 27,88 & 95 & 91,34 & 6 & 5,76 & 1 & 0,96 & 7 & 6,72 \\
\hline 2 & $\begin{array}{l}\text { I am a good } \\
\text { reader }\end{array}$ & 36 & $\begin{array}{c}34,6 \\
1\end{array}$ & 47 & 45,19 & 83 & 79,8 & 16 & 15,38 & 7 & 6,73 & 23 & 22,11 \\
\hline 3 & $\begin{array}{l}\text { I learn more } \\
\text { from reading } \\
\text { than most } \\
\text { students in the } \\
\text { class }\end{array}$ & 17 & $\begin{array}{c}16,3 \\
4\end{array}$ & 35 & 33,65 & 52 & 49,99 & 32 & 30,76 & 18 & $\begin{array}{c}17,3 \\
0\end{array}$ & 50 & 48,06 \\
\hline
\end{tabular}

Table 2 Reading Challenge

\begin{tabular}{|c|c|c|c|c|c|c|c|c|c|c|c|c|c|}
\hline \multirow[b]{2}{*}{ No } & \multirow[b]{2}{*}{ Statement } & \multicolumn{2}{|c|}{4} & \multicolumn{2}{|r|}{3} & \multicolumn{2}{|c|}{ Total } & \multicolumn{2}{|r|}{2} & \multicolumn{2}{|c|}{1} & \multicolumn{2}{|r|}{ Total } \\
\hline & & $\mathrm{F}$ & $\%$ & $\mathrm{~F}$ & $\%$ & $\mathbf{F}$ & $\%$ & $\mathrm{~F}$ & $\%$ & $\mathrm{~F}$ & $\%$ & $\mathbf{F}$ & $\%$ \\
\hline 4 & $\begin{array}{l}\text { I like hard } \\
\text { challenging } \\
\text { books }\end{array}$ & 38 & $\begin{array}{c}36,5 \\
3\end{array}$ & 24 & 23,07 & 62 & 59,6 & 3 & 2,88 & 14 & $\begin{array}{c}13,4 \\
6\end{array}$ & 17 & 16,34 \\
\hline $\begin{array}{rr}5 & \mathrm{i} \\
& 1 \\
& 1 \\
& 1\end{array}$ & $\begin{array}{l}\text { If the project is } \\
\text { interesting, I can } \\
\text { read difficult } \\
\text { material }\end{array}$ & 24 & $\begin{array}{c}23,0 \\
7\end{array}$ & 32 & 30,76 & 56 & 53,83 & 27 & 25,96 & 21 & $\begin{array}{c}20,1 \\
9\end{array}$ & 48 & 46,15 \\
\hline 6 & $\begin{array}{l}\text { I like it when } \\
\text { the questions } \\
\text { in books make } \\
\text { me think }\end{array}$ & 51 & $\begin{array}{c}49,0 \\
3\end{array}$ & 30 & 28,84 & 81 & 77,87 & 16 & 15,38 & 6 & 5,76 & 22 & 21,14 \\
\hline 7 & $\begin{array}{l}\text { I usually } \\
\text { learn difficult } \\
\text { things by }\end{array}$ & 29 & $\begin{array}{c}27,8 \\
8\end{array}$ & 35 & 33,65 & 64 & 61,53 & 21 & $\begin{array}{c}20,1 \\
9\end{array}$ & 17 & 16,34 & 38 & 36,53 \\
\hline
\end{tabular}


Jadila: Journal of Development and Innovation

reading

8 If a book is interesting I $\begin{array}{llll}26 & 25 & 35 & 33,65\end{array}$

$61 \quad 58,65 \quad 23$ $\begin{array}{llll}22,1 & 17 & 16,3 & \mathbf{4 0}\end{array}$

38,45 don't care how hard it is to read

Table 3 Reading Curiosity

\begin{tabular}{|c|c|c|c|c|c|c|c|c|c|c|c|c|c|}
\hline \multirow[b]{2}{*}{ No } & \multirow[b]{2}{*}{ Statement } & \multicolumn{2}{|c|}{4} & \multicolumn{2}{|r|}{3} & \multicolumn{2}{|c|}{ Total } & \multicolumn{2}{|r|}{2} & \multicolumn{2}{|r|}{1} & \multicolumn{2}{|r|}{ Total } \\
\hline & & $\mathrm{F}$ & $\%$ & $\bar{F}$ & $\%$ & $\mathbf{F}$ & $\%$ & $\mathrm{~F}$ & $\%$ & $\mathrm{~F}$ & $\%$ & $\mathbf{F}$ & $\%$ \\
\hline 9 & $\begin{array}{l}\text { If the teacher } \\
\text { discuss } \\
\text { something } \\
\text { interesting I } \\
\text { might read more } \\
\text { about it }\end{array}$ & 50 & $\begin{array}{c}48,0 \\
7\end{array}$ & 27 & 25,96 & 77 & 74,03 & 13 & 12,5 & 14 & $\begin{array}{c}13,4 \\
6\end{array}$ & 27 & 25,96 \\
\hline 10 & $\begin{array}{l}\text { I have favorite } \\
\text { subjects that I } \\
\text { like to read } \\
\text { about }\end{array}$ & 49 & $\begin{array}{c}47,1 \\
1\end{array}$ & 23 & 22,11 & 72 & 69,22 & 16 & 15,38 & 4 & 3,84 & 20 & 19,22 \\
\hline 11 & $\begin{array}{l}\text { I read to learn } \\
\text { new } \\
\text { information } \\
\text { about topics } \\
\text { that interest } \\
\text { me }\end{array}$ & 42 & $\begin{array}{c}40,3 \\
8\end{array}$ & 34 & 32,69 & 76 & 73,07 & 17 & 16,34 & 11 & $\begin{array}{l}10,5 \\
7\end{array}$ & 28 & 26,91 \\
\hline 12 & $\begin{array}{l}\text { I read about } \\
\text { my hobbies } \\
\text { to learn more } \\
\text { about them }\end{array}$ & 40 & $\begin{array}{c}38,4 \\
6\end{array}$ & 23 & 22,11 & 63 & 60,57 & 19 & $\begin{array}{l}18,2 \\
6\end{array}$ & 20 & 19,23 & 39 & 37,49 \\
\hline & $\begin{array}{l}\text { I like to read } \\
\text { about new thing }\end{array}$ & 51 & $\begin{array}{c}49,0 \\
3 \\
\end{array}$ & 22 & 21,15 & 73 & 70,18 & 15 & 14,42 & 13 & 12,5 & 28 & 26,92 \\
\hline & $\begin{array}{l}\text { I enjoy reading } \\
\text { books about } \\
\text { living things }\end{array}$ & 41 & $\begin{array}{c}39,4 \\
2\end{array}$ & 28 & 26,92 & 69 & 66,34 & 22 & 21,15 & 13 & 12,5 & 35 & 33,65 \\
\hline
\end{tabular}

Table 4 Reading Involvement 


\begin{tabular}{|c|c|c|c|c|c|c|c|c|c|c|c|c|c|}
\hline \multirow[b]{2}{*}{ No } & \multirow[b]{2}{*}{ Statement } & \multicolumn{2}{|r|}{4} & \multicolumn{2}{|r|}{3} & \multicolumn{2}{|r|}{ Total } & \multicolumn{2}{|r|}{2} & \multicolumn{2}{|c|}{1} & \multicolumn{2}{|r|}{ Total } \\
\hline & & $\mathrm{F}$ & $\%$ & $\mathrm{~F}$ & $\%$ & $\mathbf{F}$ & $\%$ & $\mathrm{~F}$ & $\%$ & $\mathrm{~F}$ & $\%$ & $\mathbf{F}$ & $\%$ \\
\hline 15 & $\begin{array}{l}\text { I read story } \\
\text { about fantasy } \\
\text { and make } \\
\text { believe }\end{array}$ & 19 & 18,26 & 36 & 34,61 & 55 & 52,87 & 21 & $\begin{array}{l}20,1 \\
9\end{array}$ & 23 & $\begin{array}{c}22,1 \\
1\end{array}$ & 44 & 42,3 \\
\hline & I like mysteries & & & & & & & & & & & & \\
\hline 16 & & 45 & $\begin{array}{c}43,2 \\
6\end{array}$ & 25 & 24,03 & 70 & 67,29 & 17 & 16,34 & 7 & 6,73 & 24 & 23,07 \\
\hline 17 & $\begin{array}{l}\text { I make } \\
\text { pictures in my } \\
\text { mind when I } \\
\text { read }\end{array}$ & 30 & $\begin{array}{c}16,3 \\
4\end{array}$ & 36 & 34,61 & 66 & 50,95 & 20 & 19,23 & 16 & $\begin{array}{c}15,3 \\
8\end{array}$ & 36 & 34,61 \\
\hline & $\begin{array}{l}\text { I feel like I } \\
\text { make } \\
\text { friends with } \\
\text { people in good } \\
\text { books }\end{array}$ & 22 & $\begin{array}{c}21,1 \\
5\end{array}$ & 31 & 29,80 & 53 & 50,95 & 28 & $\begin{array}{l}26,9 \\
2\end{array}$ & 23 & 22,11 & 51 & $\begin{array}{l}49,0 \\
3\end{array}$ \\
\hline 19 & $\begin{array}{l}\text { I read a lot of } \\
\text { adventure } \\
\text { stories }\end{array}$ & 43 & $\begin{array}{c}41,3 \\
4\end{array}$ & 27 & 25,96 & 70 & 67,3 & 19 & 18,26 & 14 & $\begin{array}{c}13,4 \\
6\end{array}$ & 33 & 31,72 \\
\hline 20 & $\begin{array}{l}\text { I enjoy a long, } \\
\text { involved story } \\
\text { or fiction book }\end{array}$ & 31 & $\begin{array}{l}29,8 \\
0 \\
\end{array}$ & 38 & 36,53 & 69 & 66,33 & 21 & 20,19 & 14 & $\begin{array}{l}13,4 \\
6 \\
\end{array}$ & 35 & 33,65 \\
\hline & Table 5 In & npor & tance 0 & f Rer & ding & & & & & & & & \\
\hline & & & 4 & & 3 & & Total & & 2 & & 1 & & Total \\
\hline No & Statement & $\mathrm{F}$ & $\%$ & $\mathrm{~F}$ & $\%$ & $\mathbf{F}$ & $\%$ & $\bar{F}$ & $\%$ & $\mathrm{~F}$ & $\%$ & $\mathbf{F}$ & $\%$ \\
\hline 21 & $\begin{array}{l}\text { It is very } \\
\text { important to me } \\
\text { to be a good } \\
\text { reader }\end{array}$ & 47 & $\begin{array}{c}45,1 \\
9\end{array}$ & 29 & 27,88 & 76 & 73,07 & 18 & 17,30 & 10 & 9,61 & 28 & 26,91 \\
\hline 22 & $\begin{array}{l}\text { In comparison } \\
\text { to other } \\
\text { activities I do, it } \\
\text { is very } \\
\text { important to me } \\
\text { to be a good } \\
\text { reader }\end{array}$ & 34 & $\begin{array}{c}32,6 \\
9\end{array}$ & 43 & 41,34 & 77 & 74,03 & 17 & 16,34 & 10 & 9,61 & 27 & 25,96 \\
\hline
\end{tabular}

Table 6 Reading Work Avoidance

\begin{tabular}{|c|c|c|c|c|c|c|c|c|c|c|c|c|}
\hline \multirow[b]{2}{*}{ No } & \multirow[b]{2}{*}{ Statement } & \multicolumn{2}{|c|}{4} & \multicolumn{2}{|c|}{3} & \multicolumn{2}{|c|}{ Total } & \multicolumn{2}{|c|}{2} & \multicolumn{2}{|c|}{1} & Total \\
\hline & & $\mathrm{F}$ & $\%$ & $\mathrm{~F}$ & $\%$ & $\mathbf{F}$ & $\%$ & $\mathrm{~F}$ & $\%$ & $\mathrm{~F}$ & $\%$ & $\%$ \\
\hline
\end{tabular}


Jadila: Journal of Development and Innovation

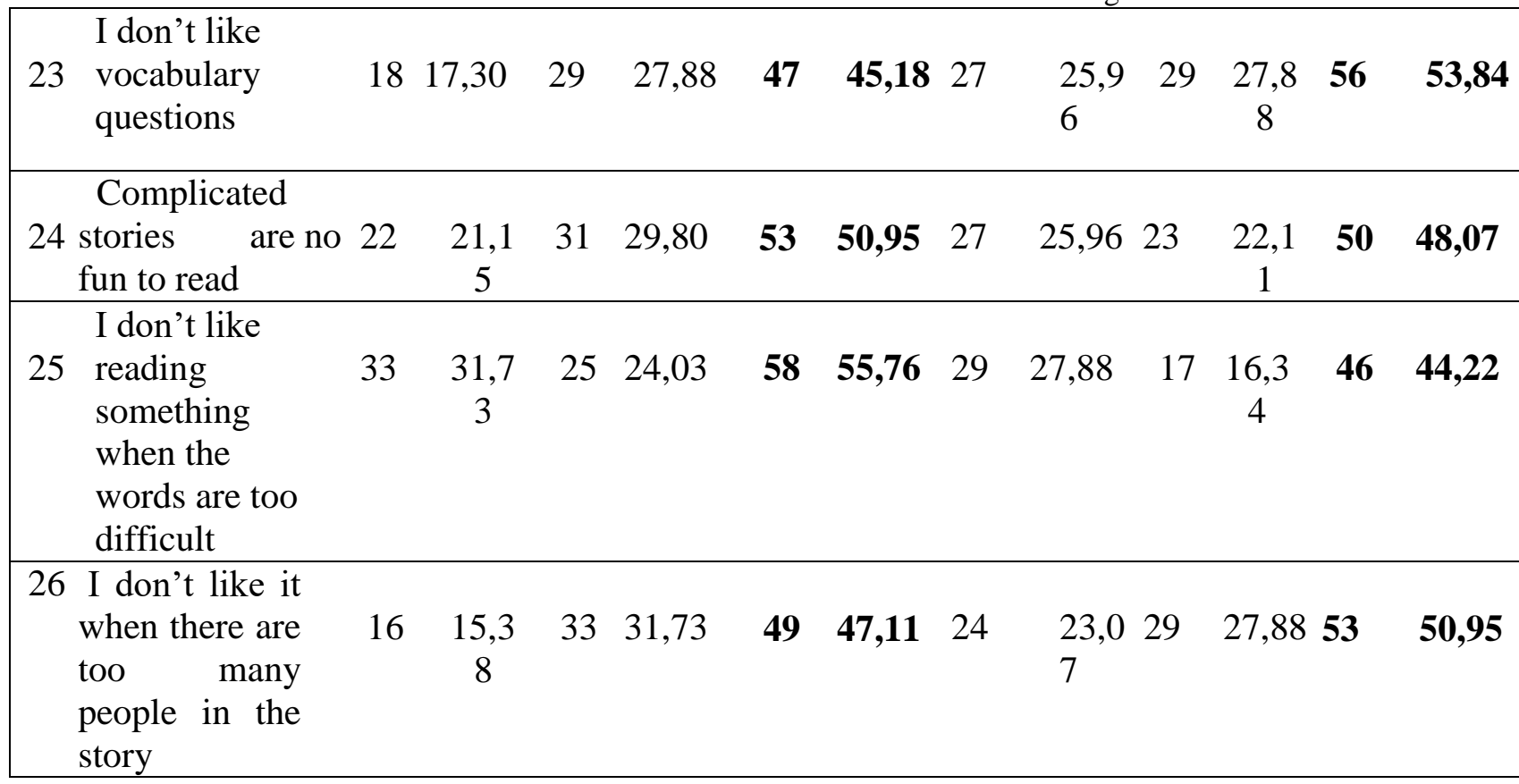

Table 7 Competition Reading

\begin{tabular}{|c|c|c|c|c|c|c|c|c|c|c|c|c|c|}
\hline \multirow[b]{2}{*}{ No } & \multirow[b]{2}{*}{ Statement } & \multicolumn{2}{|c|}{4} & \multicolumn{2}{|r|}{3} & \multicolumn{2}{|c|}{ Total } & \multicolumn{2}{|r|}{2} & \multicolumn{2}{|r|}{1} & \multicolumn{2}{|r|}{ Total } \\
\hline & & $\mathrm{F}$ & $\%$ & $\mathrm{~F}$ & $\%$ & $\mathbf{F}$ & $\%$ & $\bar{F}$ & $\%$ & $\mathrm{~F}$ & $\%$ & $\mathbf{F}$ & $\%$ \\
\hline 27 & $\begin{array}{l}\text { I try to get more } \\
\text { answer right } \\
\text { than my friends }\end{array}$ & 35 & $\begin{array}{c}33,6 \\
5\end{array}$ & 27 & 25,96 & 62 & 59,61 & 19 & $\begin{array}{c}18,2 \\
6\end{array}$ & 14 & $\begin{array}{c}13,4 \\
6\end{array}$ & 33 & 31,72 \\
\hline 28 & $\begin{array}{l}\text { I like being the } \\
\text { best at reading }\end{array}$ & 54 & $\begin{array}{c}51,9 \\
2 \\
\end{array}$ & 25 & 24,03 & 79 & 75,95 & 11 & 10,57 & 7 & 6,73 & 18 & 17,3 \\
\hline 29 & $\begin{array}{l}\text { I like to finish } \\
\text { my reading } \\
\text { before other } \\
\text { students }\end{array}$ & 33 & $\begin{array}{c}31,7 \\
3\end{array}$ & 30 & 28,84 & 63 & 60,57 & 22 & 21,15 & 18 & $\begin{array}{c}17,3 \\
0\end{array}$ & 40 & 38,45 \\
\hline 30 & $\begin{array}{l}\text { I like being } \\
\text { the only one } \\
\text { who knows } \\
\text { an answer in } \\
\text { something } \\
\text { we read }\end{array}$ & 48 & $\begin{array}{c}46,1 \\
5\end{array}$ & 22 & 21,15 & 70 & 67,3 & 19 & $\begin{array}{c}18,2 \\
6\end{array}$ & 14 & 13,46 & 33 & 31,72 \\
\hline 31 & $\begin{array}{l}\text { It is important } \\
\text { for me to see } \\
\text { my name on a } \\
\text { list of a good } \\
\text { readers }\end{array}$ & 45 & $\begin{array}{c}43,2 \\
6\end{array}$ & 31 & 29,80 & 76 & 73,06 & 13 & 12,5 & 13 & 12,52 & 26 & 25 \\
\hline
\end{tabular}


32 I am willing to work hard to read better than $43 \quad 41,3 \quad 37 \quad 35,57$

$\begin{array}{llll}80 & \mathbf{7 6 , 9 1} & 15 & 14,42\end{array}$

$17 \quad 16,3 \quad 32 \quad 30,76$ my friends

\section{Table 8 Recognition for Reading}

\begin{tabular}{|c|c|c|c|c|c|c|c|c|c|c|c|c|c|}
\hline \multirow[b]{2}{*}{ No } & \multirow[b]{2}{*}{ Statement } & \multicolumn{2}{|c|}{4} & \multicolumn{2}{|r|}{3} & \multicolumn{2}{|c|}{ Total } & \multicolumn{2}{|r|}{2} & \multicolumn{2}{|c|}{1} & \multicolumn{2}{|c|}{ Total } \\
\hline & & $\mathrm{F}$ & $\%$ & $\mathrm{~F}$ & $\%$ & $\mathbf{F}$ & $\%$ & $\mathrm{~F}$ & $\%$ & F & $\%$ & $\mathbf{F}$ & $\%$ \\
\hline 33 & $\begin{array}{l}\text { I like having the } \\
\text { teacher say I } \\
\text { read well }\end{array}$ & 38 & $\begin{array}{c}36,5 \\
3\end{array}$ & 32 & 30,76 & 70 & 67,29 & 13 & 12,5 & 17 & $\begin{array}{c}16,3 \\
4\end{array}$ & 30 & 28,84 \\
\hline 34 & $\begin{array}{l}\text { My friends } \\
\text { sometimes tell } \\
\text { me I am a good } \\
\text { reader }\end{array}$ & 25 & $\begin{array}{c}24,0 \\
3\end{array}$ & 30 & 28,84 & 55 & 52,87 & 35 & 33,65 & 11 & $\begin{array}{c}10,5 \\
7\end{array}$ & 46 & 44,22 \\
\hline 35 & $\begin{array}{l}\text { I like to get } \\
\text { compliments } \\
\text { for my } \\
\text { reading }\end{array}$ & 25 & $\begin{array}{c}24,0 \\
3\end{array}$ & 27 & 25,96 & 52 & 49,99 & 28 & 26,96 & 17 & $\begin{array}{c}16,3 \\
4\end{array}$ & 45 & 43,3 \\
\hline 36 & $\begin{array}{l}\text { I am happy } \\
\text { when } \\
\text { someone } \\
\text { recognizes } \\
\text { my reading }\end{array}$ & 37 & $\begin{array}{c}35,5 \\
7\end{array}$ & 34 & 32,69 & 71 & 68,26 & 24 & $\begin{array}{l}23,0 \\
7\end{array}$ & 8 & 7,69 & 32 & $\begin{array}{l}30,7 \\
6\end{array}$ \\
\hline 37 & $\begin{array}{l}\text { My parents } \\
\text { often tell me } \\
\text { what a good job } \\
\text { I am doing in } \\
\text { reading }\end{array}$ & 28 & $\begin{array}{c}26,9 \\
2\end{array}$ & 38 & 36,53 & 66 & 63,45 & 20 & 19,23 & 18 & $\begin{array}{c}17,3 \\
0\end{array}$ & 38 & 36,53 \\
\hline
\end{tabular}

Table 9 Reading for Grades

\begin{tabular}{|c|c|c|c|c|c|c|c|c|c|c|c|c|c|}
\hline \multirow[b]{2}{*}{ No } & \multirow[b]{2}{*}{ Statement } & \multicolumn{2}{|c|}{4} & \multicolumn{2}{|r|}{3} & \multicolumn{2}{|c|}{ Total } & \multicolumn{2}{|r|}{2} & \multicolumn{2}{|c|}{1} & \multicolumn{2}{|c|}{ Total } \\
\hline & & $\mathrm{F}$ & $\%$ & $\mathrm{~F}$ & $\%$ & $\mathbf{F}$ & $\%$ & $\mathrm{~F}$ & $\%$ & $\mathrm{~F}$ & $\%$ & $\mathbf{F}$ & $\%$ \\
\hline 38 & $\begin{array}{l}\text { Grades are good } \\
\text { way to see how } \\
\text { well you are } \\
\text { doing in reading }\end{array}$ & 37 & $\begin{array}{c}35,5 \\
7\end{array}$ & 34 & 32,69 & 71 & 68,69 & 16 & $\begin{array}{l}15,3 \\
8\end{array}$ & 13 & 12,5 & 29 & 27,88 \\
\hline 39 & $\begin{array}{l}\text { I look forward } \\
\text { to finding out } \\
\text { my reading } \\
\text { grades } \\
\end{array}$ & 32 & $\begin{array}{c}30,7 \\
6\end{array}$ & 31 & 29,80 & 63 & 60,56 & 21 & 20,19 & 15 & $\begin{array}{c}14,4 \\
2\end{array}$ & 36 & 34,61 \\
\hline 40 & $\begin{array}{l}\text { I read to } \\
\text { improve my } \\
\text { grades }\end{array}$ & 36 & $\begin{array}{c}34,6 \\
1\end{array}$ & 44 & 42,30 & 80 & 76,91 & 14 & 13,46 & 8 & 7,69 & 22 & 21,15 \\
\hline
\end{tabular}


Jadila: Journal of Development and Innovation

41 My parents ask me about my reading $32 \quad 30,7 \quad 43 \quad 41,34$ $\begin{array}{lll}75 & \mathbf{7 2 , 1} & 25\end{array}$ $\begin{array}{llll}24,0 & 13 & 12,5 & \mathbf{3 8}\end{array}$ grade

Table 10 Social Reason for Reading

\begin{tabular}{|c|c|c|c|c|c|c|c|c|c|c|c|c|c|}
\hline \multirow[b]{2}{*}{ No } & \multirow[b]{2}{*}{ Statement } & \multicolumn{2}{|c|}{4} & \multicolumn{2}{|r|}{3} & \multicolumn{2}{|c|}{ Total } & \multicolumn{2}{|r|}{2} & \multicolumn{2}{|l|}{$\mathbf{1}$} & \multicolumn{2}{|c|}{ Total } \\
\hline & & $\mathrm{F}$ & $\%$ & $\mathrm{~F}$ & $\%$ & $\mathbf{F}$ & $\%$ & $\mathrm{~F}$ & $\%$ & $\mathrm{~F}$ & $\%$ & $\mathbf{F}$ & $\%$ \\
\hline 42 & $\begin{array}{l}\text { I visit the library } \\
\text { often with my } \\
\text { family }\end{array}$ & 22 & $\begin{array}{c}21,1 \\
5\end{array}$ & 20 & 19,23 & 42 & 40,38 & 27 & $\begin{array}{l}25,9 \\
6\end{array}$ & 37 & $\begin{array}{c}35,5 \\
7\end{array}$ & 64 & 61,57 \\
\hline 43 & $\begin{array}{l}\text { I often read to } \\
\text { my brother or } \\
\text { my sister }\end{array}$ & 22 & $\begin{array}{c}21,1 \\
5\end{array}$ & 25 & 24,03 & 47 & 45,18 & 26 & 25 & 33 & $\begin{array}{c}31,7 \\
3\end{array}$ & 59 & 56,73 \\
\hline 44 & $\begin{array}{l}\text { My friends } \\
\text { and I like to } \\
\text { trade things to } \\
\text { read }\end{array}$ & 35 & $\begin{array}{c}33,6 \\
5\end{array}$ & 20 & 19,23 & 55 & 52,88 & 22 & 21,15 & 27 & $\begin{array}{c}25,9 \\
6\end{array}$ & 49 & 47,11 \\
\hline 45 & $\begin{array}{l}\text { I sometimes } \\
\text { read to my } \\
\text { parents }\end{array}$ & 17 & $\begin{array}{c}16,3 \\
4 \\
\end{array}$ & 28 & 26,92 & 45 & 43,26 & 20 & $\begin{array}{l}19,2 \\
3\end{array}$ & 36 & 34,61 & 56 & $\begin{array}{l}53,8 \\
4 \\
\end{array}$ \\
\hline 46 & $\begin{array}{l}\text { I talk my } \\
\text { friends about } \\
\text { what I am } \\
\text { reading }\end{array}$ & 36 & $\begin{array}{c}34,6 \\
1\end{array}$ & 28 & 26,92 & 64 & 61,53 & 23 & $\begin{array}{l}22,1 \\
1\end{array}$ & 20 & $\begin{array}{c}19,2 \\
3\end{array}$ & 43 & 41,34 \\
\hline 47 & $\begin{array}{l}\text { I like to help } \\
\text { my friends } \\
\text { with their } \\
\text { schoolwork in } \\
\text { reading }\end{array}$ & 38 & $\begin{array}{c}36,5 \\
3\end{array}$ & 32 & 30,76 & 70 & 67,29 & 18 & 17,30 & 15 & $\begin{array}{c}14,4 \\
2\end{array}$ & 33 & 31,72 \\
\hline 48 & $\begin{array}{l}\text { I like to tell my } \\
\text { family about } \\
\text { what I am } \\
\text { reading }\end{array}$ & 19 & $\begin{array}{c}18,2 \\
6\end{array}$ & 35 & 33,65 & 54 & 51,91 & 28 & 26,92 & 22 & $\begin{array}{c}21,1 \\
5\end{array}$ & 50 & 48,07 \\
\hline
\end{tabular}

\section{Discussion}

After analyzing the data by distributing questionnaires, it can be concluded that there were three factors that influenced students' reading motivation of SMP N 7 Pemulutan.

The first concerning self-efficacy was represented by two dimensions namely, reading efficacy and reading challenge. According to Bandura (1994) self-efficacy has been described as one's perceived abilities at specified levels. As the result, students believe they should develop 
Jadila: Journal of Development and Innovation

E-ISSN: 2723-6900

in Language and Literature Education

P-ISSN: 2745-9578

Publisher: Yayasan Karinosseff Muda Indonesi

Volume 1 Number 2, 2021

Page: $88-100$

reading skills for reading effectiveness, they learn more reading and the students were inspired to read books to be a successful reader. Khairuddin (2000) explained that in school and life, reading was the road to success, one who did want to learn would not be able to succeed. In short, reading was very influence in people daily life especially for students.

The second, students were also proved to have intrinsic motivation, for reading as they have highly percentage in the statements composing the four dimensions namely, reading curiosity, reading involvement, reading work avoidance, importance of reading. As the result, four indicators only three were the most dominant for students, except reading work avoidance. According to Deci (1975) intrinsically motivated behaviors were activities that have no obvious reward except for the activity itself.

The third was presented extrinsic motivation and social motivation for reading. The students were more extrinsically motivated, seen from the frequency of answering items most of them choose to agree with the statement. Wang \& Guthrie (2004) described that, extrinsic motivation comes from outside where an person tries to fulfill "external values and demands". Included five dimension namely, reading recognition, reading for grades, competition in reading, social reason in reading, and compliance.

\section{Conclusion}

Regarding to the previous findings and discussion. It can be concluded that the students reading motivation in English at SMP N 7 Pemulutan was good. The eleven dimensions of reading motivation have all proved to be present in the students who participated in the study. The survey covers (a) self-efficacy factors can make the students more motivated in reading. (b) Intrinsic factors included enjoyable, and students' interest in reading. (c) Extrinsic factors covered such as for finishing assignment.

\section{References}

Alhamdu. (2016). Interest and Reading motivation. International Journal of Linguistics IV (4). http://www.researchgate.net/publication/323576174 Anderson, R. C. Becomin a nation a reader of the commision on reading prepared. 
Arnold, J. (2000). Affect in language learning. Cambridge: Cambridge University Press.

Bandura, A. (1994). Self-Efficacy. Enyclopedia of mental health , 4. http://www.researchgate.net//279616365

Brophy, J. (2004). Motivating student to learn (Second Edition ed.). London, new jersey: Lawrence ErlBaum Associates.

Creswell, J. W. (2012). Educational Research (Fourth Edition ed.). (P. c. corp, Ed.) Nebraska: Pearson Education.

Dakhi, S., \& Damanik, I. S. (2018). Students motivation in reading English text : a qualitative study EFL context. Journal of English Teaching , 4 (2), 81-93. https://www.researchgate.net/publication/327860525

Deci, E. L. (1975). Intrinsic Motivation. (E. Aronson, Ed.) New York: Plenum press London.

Deci, L. E., \& Ryan, R. M. (2000). Facilitating Optimal Motivation and Psychological Well-Being Across Life's Domains. Canadian Psychology, 49.

Dornyei, Z. (2001). Motivational Strategies in the Language Classroom. New york: Cambridge University Press.

Guthrie, J. T., Wiegfield, A., \& Perencevich, K. C. (2010). Motivating Reading Comprehension. New York: 10 industrial Avenue and Routledge.

Houghton, K. L. (2015, December). Impact of Intrinsic and Extrinsic Motivation on Reading Achievment of First Grade Students. Walden University .

Jhonson, R. B., \& Christensen, L. (2014). Educational Research (5 Edition ed.). (R. Hester, Ed.) United stated, America: Sage Publications, Inc.

Khairuddin, Z. (2013). A Study of Students' Reading Interest in Second Language. International Education Studies, VI (11), 160.

kusumaningputri, r. (2014). indonesian learners motivation in english reading classrom. 4 (2). http://journal.ubm.ac.id/index.php/english language_culture/article/download/325/312

Marhama, N. A. (2013). A srvey on student motivation in Reading English text. http://repository.ung.ac.id/skripsi/show/321408078/a_survey on students motivation in reading_English text.html

Mendil, i. (2016). lack of reading motivation in the algeria EFL classroom.

McGeown, S. P. (2013). Reading Motivation and engagement in the primary school classroom: theory, research and practice (Vol. X). (R. Levy, Ed.) United Kingdom: UKLA.

Mehdi. G (2006). Reading Motivation in Students' of English as a Foreign Language(Thesis).RetrievedFrom.https://bu.umc.edu.dz/theses/anglais /GRI1048.PDF

pang, E. s., Muaka, A., Bernbart, E. B., \& Kamil, M. L. (2003). Teaching Reading. Educational Practice series 12.

Patton, M. Q. (1980). Qualitative Evaluation methods. Beverly Hills, California: SAGE publications. 
Ryan, R. M., \& Deci, E. L. (2000). Self-Determination theory and the facilitation of intrinsic motivation social development and well being. 55 (1), 68-78.

Schraw, G., \& Lehman, S. (2001). Situational Interest: A review of the Litrature and Direction for Future Research (Vol. 13). Educational Psychology Review.

Serin, H. (2018). The Use of Extrinsic and Intrinsic Motivations to Enhance StudentAchievement in Educational Setting. International Journal of Social Sciences \& Educational Studies, V(1).

Siregar, A. I.-R., \& Narius, D. (2016). An Analysis of Students Reading Motivation in Reading Subject at English Department of Universitas Negeri Padang. Journal of English Language Teaching, Viii (3).

Wigfield, A., \& Guthrie, J. T. (1997). Relations of Childrens' Motivation for Reading to the Amount and Breadth of Their Reading. Journal of Educational Psychology, LXXXIX (3), 420-432. 